\title{
Avaliação Ambiental Estratégica orientada pela transdisciplinaridade
}

\author{
Transdisciplinarity-oriented Strategic Environmental Assessment
}

Antonio Waldimir Leopoldino da Silva', Paulo Maurício Selig ${ }^{2}$

口-

\begin{abstract}
RESUMO
Situada na interface entre ciência, política e sociedade, a Avaliação Ambiental Estratégica (AAE) envolve múltiplas áreas de saber e questões sujeitas a juízos de valor. Este trabalho visou analisar a interrelação entre perfil transdisciplinar da AAE, conhecimento local (CL), participação do público e efetividade da avaliação. A AAE orientada à transdisciplinaridade (AAEt) permite melhor abordagem do contexto em estudo, por integrar concepções e perspectivas de agentes com diferentes formações, saberes, experiências e visões de mundo. O CL atua como um conhecimento contextual, que complementa o conhecimento científico. A AAEt deve pautar-se por uma política de envolvimento continuado entre cientistas e leigos, por meio da participação colaborativa de atores sociais. Em conjunto, esses elementos levam à maior aceitação dos resultados pelos interessados, aumentando a efetividade da ferramenta.
\end{abstract}

Palavras-chave: Avaliação Ambiental Estratégica; conhecimento local; efetividade; processos participativos; transdisciplinaridade.

\begin{abstract}
Located at the interface between science, politics and society, Strategic Environmental Assessment (SEA) involves multiple knowledge areas and issues subject to value judgments. This study aims to analyze the interrelation between transdisciplinary profile of SEA, local knowledge (LK), public engagement and effectiveness of assessment. Transdisciplinarity-oriented SEA (SEAt) allows better approach of the context under study, because it integrates concepts and perspectives of agents with different backgrounds, knowledge, experiences and worldviews. The LK acts as a contextual knowledge and it complements the scientific knowledge. SEAt should be guided by a policy of continued involvement between scientists and lay people, through collaborative participation of social actors. Together, these factors lead to greater acceptance of the results by stakeholders, increasing the tool's effectiveness.
\end{abstract}

Keywords: Strategic Environmental Assessment; local knowledge; effectiveness; participative processes; transdisciplinarity.

\section{INTRODUÇÃO}

Ferramenta de crescente importância no cenário internacional, a Avaliação Ambiental Estratégica (AAE) tem por objetivo integrar considerações ambientais e de sustentabilidade à tomada de decisão estratégica, seja no âmbito de políticas, planos ou programas (THERIVEL, 2010). Nesse âmbito, a AAE configura-se como um processo de produção e sistematização de conhecimentos sobre o contexto em análise, envolvendo questões complexas, multifacetadas e sujeitas a juízos de valor. A prática da avaliação deve, portanto, atender a duas premissas

fundamentais: adoção de uma abordagem que combine múltiplas áreas de saber - tais como ciências naturais, exatas, humanas e sociais a fim de retratar e projetar toda a abrangência do objeto estudado; e incorporação de valores, percepções, opiniões e conhecimentos dos atores sociais, aprimorando o processo e fazendo com que seus resultados sejam legitimados pelos segmentos envolvidos.

Runhaar (2009) destaca que a aceitação de uma investigação ambiental é aumentada mediante a participação das partes interessadas, pois permite que estas contribuam com ideias, conhecimentos

\footnotetext{
口-

${ }^{1}$ Engenheiro Agrônomo. Mestre em Zootecnia. Doutor em Engenharia e Gestão do Conhecimento. Professor Efetivo da Universidade do Estado de Santa Catarina (UDESC) Chapecó (SC), Brasil.

2Doutor em Engenharia de Produção. Professor do Programa de Pós-Graduação em Engenharia e Gestão do Conhecimento da Universidade Federal de Santa Catarina (UFSC) - Florianópolis (SC), Brasil.

Endereço para correspondência: Antonio Waldimir Leopoldino da Silva - UDESC Chapecó - Rua Beloni Trombetta Zanin, 680E - Santo Antônio - 89815-630 - Chapecó (SC), Brasil - E-mail: awls12@hotmail.com

Recebido: 11/12/2012 - Aceito: 05/04/2013 - Reg. ABES: 108213
} 
e prioridades. Bonifazi et al. (2011) salientam que um maior envolvimento da comunidade na AAE pode melhorar a base cognitiva e ética sobre a qual as políticas são formuladas, havendo a necessidade da interação entre especialistas, formuladores de política e cidadãos em sistemas de coprodução. Tais pressupostos conduzem a uma AAE que seja estruturada sob uma concepção supradisciplinar e participativa, isto é, adequada à natureza transdisciplinar dos processos de tomada de decisão (SHEATE \& PARTIDÁRIO, 2010). No entanto, em muitos casos esses aspectos não são observados e a AAE acaba por não cumprir o seu papel. Também no campo teórico os temas transdisciplinaridade (TD) e conhecimento local (CL) merecem maior atenção da literatura científica relacionada à AAE. Comprovando a existência de tal lacuna, busca realizada no dia 05 de abril de 2013 nas bases de dados Scopus e Web of Science, empregando os descritores Strategic Environmental Assessment e transdisciplinary (ou transdisciplinarity), nos campos título, resumo e palavras-chave, sem restrição de tipo de material, retornou sem qualquer indicação de publicação.

Este trabalho objetivou analisar a inter-relação existente entre

1. efetividade da AAE,

2. seu desejável ou necessário caráter transdisciplinar,

3. o emprego de CL visando alcançá-lo e

4. a participação de atores sociais como fator de viabilização dos aspectos anteriores.

\section{METODOLOGIA}

Diante da lacuna da literatura científica, antes apontada, o presente estudo constitui uma pesquisa bibliográfica que procurou estabelecer uma visão panorâmica da questão em análise. A pesquisa bibliográfica consiste na (re)construção do conhecimento a partir da consulta e análise a fontes bibliográficas. Cabe salientar, no entanto, que, segundo Marconi e Lakatos (2010, p. 166),

a pesquisa bibliográfica não é mera repetição do que já foi dito ou escrito sobre certo assunto, mas propicia o exame de um tema sob novo enfoque ou abordagem, chegando a conclusões inovadoras.

Moreira e Caleffe (2008) alertam que a pesquisa bibliográfica não deve ser confundida com revisão bibliográfica, a qual é um componente obrigatório de todo e qualquer tipo de pesquisa. Enquanto a revisão busca expor o estado da arte do conhecimento sobre um dado tema, a pesquisa bibliográfica é uma metodologia que se presta a "formular problemas" e a "encontrar respostas", ou seja, por meio dela são buscadas futuras questões de pesquisa e/ou possíveis respostas, soluções e encaminhamentos para os problemas já constatados.

\section{RESULTADOS E DISCUSSÃO}

\section{Efetividade da Avaliação Ambiental Estratégica vista como aceitação pelas partes interessadas}

Um importante elemento para a consolidação e expansão da AAE no cenário mundial é a sua efetividade como ferramenta de política ambiental. Ainda que a questão admita uma pluralidade de interpretações, há um claro direcionamento no sentido de entender que uma AAE é tanto mais efetiva e exitosa quanto maior a influência que exerce sobre o processo de tomada de decisão (STOEGLEHNER et al., 2009; VAN DOREN et al., 2013). "O sucesso da AAE deve ser medido em relação à qualidade da decisão final e ao quanto a decisão foi melhorada como resultado da abordagem da AAE" (PARTIDÁRIO, 2000, p. 658).

Para ser eficaz, a avaliação ambiental deve apresentar relevância política, validade e credibilidade científica e legitimidade às partes interessadas (NILSSON et al., 2005). Stoeglehner et al. (2009) explicam que a avaliação da efetividade depende das necessidades e expectativas - possivelmente conflitantes - dos diferentes agentes envolvidos na AAE. "A prática tem mostrado que uma AAE efetiva não é apenas sobre ciência, mas também sobre valores" (PARTIDÁRIO, 2000, p. 659). Runhaar (2009) e Runhaar e Driessen (2007) indicam que a racionalização da tomada de decisão não requer apenas um conhecimento válido cientificamente, mas também um conhecimento que seja aceito pelos vários grupos de atores.

Para Fischer (2005), se os resultados da AAE não atenderem aos valores dos tomadores de decisão, partes interessadas e outros envolvidos, sua implantação será bastante difícil, senão impossível, mesmo diante de uma documentação de qualidade. Assim, a participação pública (PP) e/ou de interessados é um dos fatores mais referidos pela literatura entre aqueles que determinam a efetividade da AAE (RUNHAAR, 2009; RUNHAAR \& DRIESSEN, 2007; VAN DOREN et al., 2013). Van Buuren e Nooteboom (2009) descrevem que a efetividade da AAE está relacionada não só ao uso do conhecimento para conduzir a decisões racionais e sustentáveis, mas também à sua contribuição para um diálogo colaborativo entre as partes interessadas. Gauthier et al. (2011) defendem que os membros leigos exerçam um papel maior do que apenas gerar informações aos especialistas, devendo ser chamados a construir soluções negociadas que levem a políticas, planos e programas (PPP) desenvolvidos de forma colaborativa. Adotando esse perfil, a AAE sediará um processo de aprendizagem mútua entre atores de diferentes percepções, resultando em um corpo de conhecimento construído conjuntamente (VAN BUUREN \& NOOTEBOOM, 2010). 


\section{Transdisciplinaridade: uma diretriz para Avaliação Ambiental Estratégica}

Um dos maiores desafios da AAE é definir e aplicar princípios e procedimentos que levem à ampliação de sua efetividade. Considerando que estão envolvidos múltiplos valores e diferentes sistemas de conhecimento, a compatibilização ou harmonização das visões que integram o processo passa a ser tão importante quanto as conclusões técnicas produzidas. Parte-se da conjectura de que a adoção de um desenho conceitual e metodológico fundamentado na TD - abordagem dialógica por natureza - pode representar um diferencial qualitativo à ferramenta, aumentando seu grau de resolutividade. Conforme mostra a Figura 1, a efetividade da AAE depende da aceitação pelas partes interessadas e mantém relação direta com a participação colaborativa de atores sociais, com o CL ou leigo e com o perfil transdisciplinar do processo.

Antes de abordar a aplicação da TD e do CL em avaliações ambientais, cabe fazer um breve resgate teórico e conceitual desses elementos.

\section{O significado epistemológico da transdisciplinaridade}

Grande parte da literatura acadêmica distingue as abordagens supradisciplinares - multidisciplinaridade (MD), interdisciplinaridade (ID) e TD - tomando por referência o nível de integração entre as disciplinas envolvidas. Nesse sentido, a MD caracteriza-se por promover a associação de diferentes pontos de vista, mas com objetivos disciplinares específicos e distintos. Os agentes trocam conhecimento, mas não tencionam cruzar fronteiras para criar um saber novo e integrativo, ou seja, as ações são paralelas, mas não integradas (TRESS et al., 2004). A ID é definida pela forte integração entre disciplinas, que extrapolam seus limites e associam seus conceitos, modelos e métodos (UITERKAMP \& VLEK, 2007;

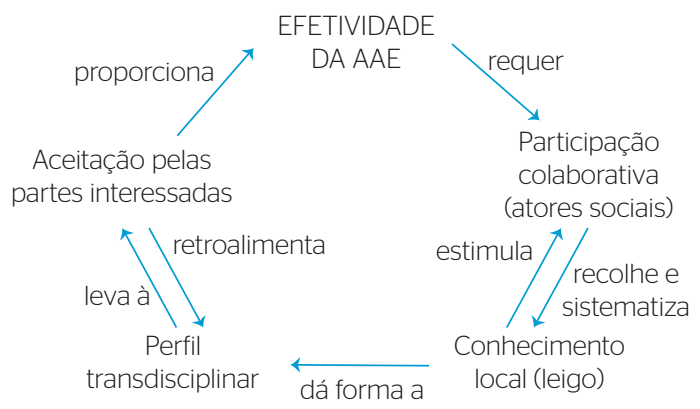

AAE: Avaliação Ambiental Estratégica.

Figura 1 - Possível relação entre efetividade da Avaliação Ambiental Estratégica, participação colaborativa de atores sociais, conhecimento local (leigo), perfil transdisciplinar e aceitação pelas partes interessadas.
MOBJÖRK, 2010). No caso, verifica-se a criação de um novo conhecimento ou teoria, ou até mesmo a emergência de uma nova disciplina, sem que seja possível isolar os componentes disciplinares originais (TRESS et al., 2004).

Todavia, a distinção entre as abordagens supradisciplinares não se limita ao grau (amplitude) de integração e colaboração entre as disciplinas, estando relacionada também aos tipos de atores e conhecimentos envolvidos. Sob esse enfoque, enquanto MD e ID envolvem apenas o meio acadêmico/científico, a TD pressupõe a participação das partes interessadas e do público comum, representando a integração entre conhecimento científico e conhecimento tradicional, local, leigo ou cidadão (LUKS \& SIEBENHÜNER, 2007; MOBJÖRK, 2010; POHL, 2008; STOCK \& BURTON, 2011; TRESS et al., 2004; UITERKAMP \& VLEK, 2007).

A TD é, pois, o resultado da combinação entre a ID e uma abordagem participativa, superando as fronteiras entre as comunidades científica e não científica (TRESS et al., 2004; UITERKAMP \& VLEK, 2007). Desse modo, significa a coprodução de um conhecimento "socialmente mais robusto" pela interação entre diferentes culturas (LUKS \& SIEBENHÜNER, 2007; POHL, 2008). Mobjörk (2010) destaca que a TD pode ser classificada em consultiva e participativa. Na primeira, os atores sociais externos à academia têm tão somente o papel de responder e reagir à pesquisa, não sendo incorporados à produção de conhecimento. Já na TD participativa os membros leigos são incluídos como participantes ativos e seu conhecimento é considerado tão valioso quanto o conhecimento científico.

\section{Conhecimento local}

O conhecimento característico de territórios e próprio de suas populações recebe várias designações, tais como tradicional, leigo, local, indígena ou aborígene, que são empregadas como sinônimos ou diferenciadas entre si conforme a abordagem. Não obstante a expressão "conhecimento tradicional" ser largamente utilizada pela literatura, Failing et al. (2007) avaliam que o termo CL é mais inclusivo e descritivo. Segundo os autores, o CL está associado a três fundamentos: ser baseado na experiência e, de modo particular, na observação pessoal; apresentar caráter holístico; estar ancorado em visões e conclusões relacionadas ao tempo e ao contexto. Yli-Pelkonen e Kohl (2005) concordam que o CL é fortemente contextualizado e inclui elementos - como experiências e crenças que são resultado dos papéis dos vários atores locais em seu tempo e espaço. Para Reed (2008, p. 2425), o CL é "primariamente tácito, implícito, informal, contexto-dependente, resultante de experiência coletiva de gerações de observação e prática”.

A associação do CL à designação "tradicional" pode transmitir a noção errônea de um conhecimento estático, obsoleto e preso 
ao passado. Entretanto, há um permanente processo de interação do cidadão leigo com o ambiente, fazendo com que o CL seja aprimorado diante das sucessivas alterações biofísicas, sociais ou culturais do meio. Ou seja, o CL continuamente se renova e evolui, mantendo-se contemporâneo (FAILING et al., 2007; STEVENSON, 1996; USHER, 2000). É inegável, também, que o CL e o conhecimento científico influenciam-se mutuamente (ICSU, 2002; STEVENSON, 1996). Yli-Pelkonen e Kohl (2005) afirmam que informações locais acerca da natureza podem variar de opiniões, sensações e experiências até observações ricas em conhecimento e perspectivas bem fundamentadas, as quais se aproximam da abordagem científica.

Usher (2000, p. 192) qualifica o conhecimento ecológico tradicional como "uma forma de ciência" e explica que, mais do que observações pessoais isoladas e desconectadas, ele envolve fatos empíricos e associações baseadas em observação e na experiência acumulada, compartilhada e validada por testes em situações práticas. Croal et al. (2012, p. 1) descrevem o conhecimento tradicional:

com suas raízes no passado, ele é tanto acumulativo quanto dinâmico, baseado na experiência de gerações anteriores e adaptado às novas mudanças tecnológicas e socioeconômicas do presente.

Portanto, salvo o caso de raras populações em completo isolamento relacional, o CL não está isento da ciência e com esta sofre um contínuo e natural processo de hibridação. Nesses moldes, o CL posiciona-se como "ciência leiga".

\section{O emprego do conhecimento local em avaliações ambientais}

$\mathrm{Na}$ área da avaliação ambiental, o emprego do CL tem merecido o devido destaque. O International Council for Science - ICSU (2002, p. 19) recomenda "assegurar a plena e efetiva participação dos portadores de conhecimento tradicional durante todos os estágios de elaboração de políticas, planos e programas de desenvolvimento sustentável, ao lado da comunidade científica e tecnológica”. A Associação Internacional para Avaliação de Impactos (IAIA) defende que as populações nativas podem oferecer importantes contribuições por intermédio de seu conhecimento sobre uso e gestão do meio ambiente e de seus valores a respeito deste (CROAL et al., 2012). Entre os "princípios básicos" apresentados pela IAIA, o primeiro preconiza que "o conhecimento tradicional deve ser tratado com o mesmo respeito e validade que o conhecimento ocidental baseado na ciência" (p. 2).

Autores como Kwiatkowski et al. (2009), Sallenave (1994) e Whitfield et al. (2011), entre outros, apontam a importância de realizar a associação entre CL e conhecimento científico em avaliações que envolvam sustentabilidade e meio ambiente. Por outro lado, Booth e Skelton (2011), Ellis (2005) e Stevenson (1996) relatam as dificuldades que cercam a questão, fazendo com que, na prática, nem sempre o CL desempenhe um papel significativo nesses estudos (O'FAIRCHEALLAIGH, 2007; PACI et al., 2002).

No que tange especificamente à AAE, Runhaar e Driessen (2007, p. 5) ratificam que o conhecimento científico "tem que ser complementado com formas de 'conhecimento leigo' ou 'ciência dos cidadãos”. Para Connelly e Richardson (2005), o não emprego do CL remove do processo um importante conhecimento contextual, e, por isso, os argumentos a favor de uma AAE voltada a especialistas não se sustentam. Azcarate e Balfors (2009) verificaram que o CL propiciou melhor compreensão das relações entre aspectos ambientais e socioeconômicos da área em estudo, favorecendo a condução do processo de AAE.

Em processos de AAE, o CL pode ser empregado de forma colaborativa com o conhecimento científico em inúmeras atividades ou etapas, tais como:

- definição de indicadores de sustentabilidade a serem utilizados como elementos de mensuração dos efeitos potenciais ou reais do PPP;

- coleta e compartilhamento de dados e informações relacionados à área ou ao objeto em avaliação (AZCARATE \& BALFORS, 2009; SPENGLER, 2009);

- desenho de propostas e opções alternativas ao PPP em estudo;

- projeção de cenários e tendências, em cada uma das opções avaliadas;

- prognóstico e previsão de possíveis impactos a serem causados pelas propostas sobre o ambiente físico e humano (RAUSCHMAYER \& RISSE, 2005);

- desenvolvimento de medidas de compensação, no caso de impactos que não possam ser evitados (SPENGLER, 2009);

- descrição de tradições, crenças, valores, hábitos e costumes das populações locais, o que permitirá compreender e contemplar seus interesses, preocupações e posicionamentos.

Há de se registrar, no entanto, que a incorporação efetiva do CL não tem sido uma prática usual na AAE. Bonifazi et al. (2011), em análise de 25 processos de AAE realizados na Itália, constataram que em algumas iniciativas o público foi o coprodutor de informação, e o CL foi usado para embasar questões em áreas específicas e para sugerir possíveis soluções. Entretanto, em cerca de dois terços dos casos, o público leigo foi um simples "recipiente" da informação, ou seja, apenas ocasionalmente os cidadãos contribuíram com seu conhecimento. A tendência de a AAE subestimar o CL é também mencionada por Balducci et al. (2011). 


\section{Reflexos de uma Avaliação Ambiental Estratégica transdisciplinar}

A sustentabilidade apresenta um caráter científico "inerentemente transdisciplinar" (STOCK \& BURTON, 2011, p. 1091), o que remete à necessidade de estudá-la mediante uma abordagem que contemple tal aspecto. Seguindo esse entendimento, Wiek e Binder (2005) afirmam que a probabilidade do alcance de bons resultados será maior se as avaliações da sustentabilidade forem conduzidas por meio da TD. Os autores apontam que a TD permite a participação de interessados com diferentes pontos de vista, obrigações, habilidades e recursos, provendo uma estrutura que adapta a ferramenta às capacidades perceptivas, cognitivas e discursivas dos atores, bem como a seus valores e preferências, o que leva a decisões socialmente estáveis e cientificamente fundamentadas.

Sediada na interface entre ciência, política e sociedade, com foco no desenvolvimento sustentável, a AAE deve configurar-se como uma construção de conhecimento enraizada na TD participativa. Para adquirir esse caráter transdisciplinar, é imperativo que o processo de AAE cumpra dois requisitos fundamentais: a equipe elaboradora deve reunir especialistas das várias áreas técnicas pertinentes, provendo uma avaliação multidisciplinar mediante condução interdisciplinar (PARTIDÁRIO, 2007); a participação dos atores sociais deve ser efetivada como um procedimento metodológico contínuo, permitindo a inclusão do CL e da opinião popular no transcurso do processo de avaliação. A concretização simultânea dessas condições, contudo, constitui uma tarefa complexa e exigente em termos de tempo.

Além do resgate e da utilização do CL, o emprego de uma abordagem pautada na TD participativa aprimora a AAE em aspectos como:

- melhor e mais completa abordagem das questões em estudo, devido à integração de concepções e perspectivas de atores com diferentes formações, saberes, experiências e visões de mundo;

- incorporação e correspondente análise dos fatores intrínsecos ao contexto considerado (elementos locais), permitindo adequada problematização, assim como objetivos e soluções compatíveis com as condições biofísicas e humanas ali presentes;

- maior comprometimento dos atores locais com o desenrolar e com os resultados da avaliação, com a implantação do PPP e com o monitoramento dessa implantação, de modo a elevar os níveis de "cidadania ambiental" (BONIFAZI et al., 2011);

- democratização do processo de avaliação, possibilitando a expressão de diferentes segmentos e interesses e promovendo uma tomada de decisão colaborativa (VAN BUUREN \& NOOTEBOOM, 2010), com maior legitimidade das conclusões com a sociedade;
- ampliação de oportunidades e processos de troca de conhecimento e de aprendizagem mútua entre cientistas e leigos, levando ao empoderamento recíproco;

- em função dos fatores acima, maior acreditação, aceitação e difusão da ferramenta como elemento de salvaguarda ao desenvolvimento sustentável.

Na prática, porém, o conceito de TD e suas implicações são pouco conhecidos entre especialistas que atuam na avaliação de impactos. Bond et al. (2010) mostram que a maioria dos técnicos envolvidos em processos de Avaliação de Impacto Ambiental (AIA) não sabe distinguir $\mathrm{MD}$, ID e TD, tampouco possuem compreensão quanto ao emprego destas abordagens na ferramenta que utilizam. Os autores destacam o predomínio de arranjos multidisciplinares de conhecimento, em relação às outras formas. Já Wiek e Binder (2005) apontam que o potencial e as lições da TD não têm sido utilizados em avaliações ambientais, incluindo a AAE.

\section{Participação colaborativa de atores sociais: o caminho para a Avaliação Ambiental Estratégica transdisciplinar}

A PP é descrita como o envolvimento de indivíduos e grupos interessados ou afetados pela intervenção proposta, os quais optam por ter um papel ativo no processo, buscando que seus interesses, necessidades e valores sejam incorporados na decisão (GONZÁLEZ et al., 2008; REED, 2008). Na AAE, sua importância está ligada à mudança paradigmática ora em curso. $\mathrm{O}$ modelo racionalista e tecnocrático, centrado no conhecimento científico e na lógica linear, determinística e positivista, vem dando lugar a (ou passou a dividir espaço com) um modelo interativo, que valoriza elementos como diálogo, negociação, flexibilidade, governança, cooperação entre atores, interesses, valores e aprendizagem social (GAUTHIER et al., 2011). Tal mudança representa a passagem de uma "ciência aplicada" para uma "ciência cívica" (CASHMORE, 2004).

Embora consagrado na literatura de avaliação ambiental, o termo "PP" simboliza uma expressão do tipo "guarda-chuva", de grande amplitude semântica, mas pouco apropriada ao real conceito de TD. Isso porque participar não necessariamente traduz uma postura ativa, interativa e contributiva, podendo significar apenas "estar presente". Construir uma AAE transdisciplinar requer mais do que participação, sendo indispensável uma política de envolvimento cooperativo continuado entre cientistas e leigos, de modo a propiciar o compartilhamento de paradigmas, a simbiose de saberes e atitudes de cocriação ao longo de toda a avaliação. Por isso, mais do que participação, é preciso fazê-la colaborativa. O modelo convencional de AAE, no qual as atividades de PP são pontuais e, não raro, apenas um acessório metodológico, deve ser superado. 
Busca-se um modelo de AAE que prime pela "participação colaborativa de atores sociais", em que a TD seja o eixo norteador das ações e concepções e componha a mente e o fazer de cada participante. A finalidade do engajamento público passa a ser "a construção e o intercâmbio de conhecimento por meio de soluções criativas para os problemas identificados, ao invés de simples mecanismos de transferência de informação através de processos consultivos" (SHEATE \& PARTIDÁRIO, 2010, p. 280).

O envolvimento dos cidadãos é um fator crítico para a obtenção e incorporação do CL, e, como tal, para o alcance da TD. A Figura 2 apresenta os vários níveis de relação e interação entre público e AAE.
Do contingente total a quem a avaliação poderá afetar ou interessar, apenas uma pequena parte exercitará uma atuação verdadeiramente colaborativa, diante dos inúmeros "filtros" (barreiras) que ocorrem em processos participativos. Therivel (2010) cita a dificuldade de mobilizar o público, pois normalmente as ações estratégicas estão afastadas do cotidiano das pessoas, resultando em um baixo número de participantes. A literatura ressalta ainda que, em algumas situações, verifica-se a chamada "fadiga de participação" (REED, 2008; SHEATE \& PARTIDÁRIO, 2010; THERIVEL, 2010), a qual tem reduzido o interesse popular pelo fato de que o processo nem sempre é democrático e ambientalmente justo.

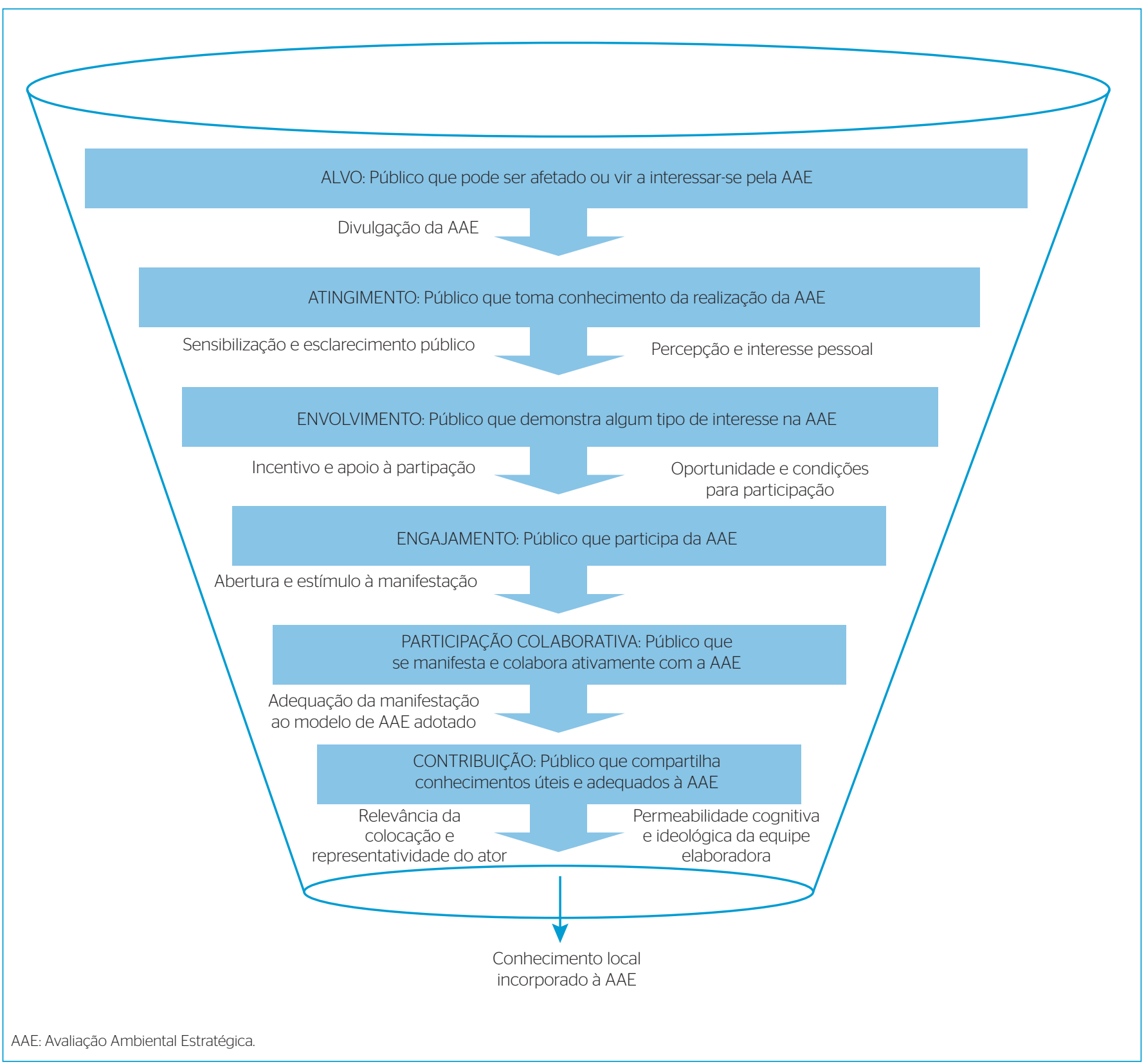

Figura 2 - Níveis de relação entre público e Avaliação Ambiental Estratégica (retângulos) e fatores que atuam como "filtros" no processo de incorporação do conhecimento local (ao lado das setas). 


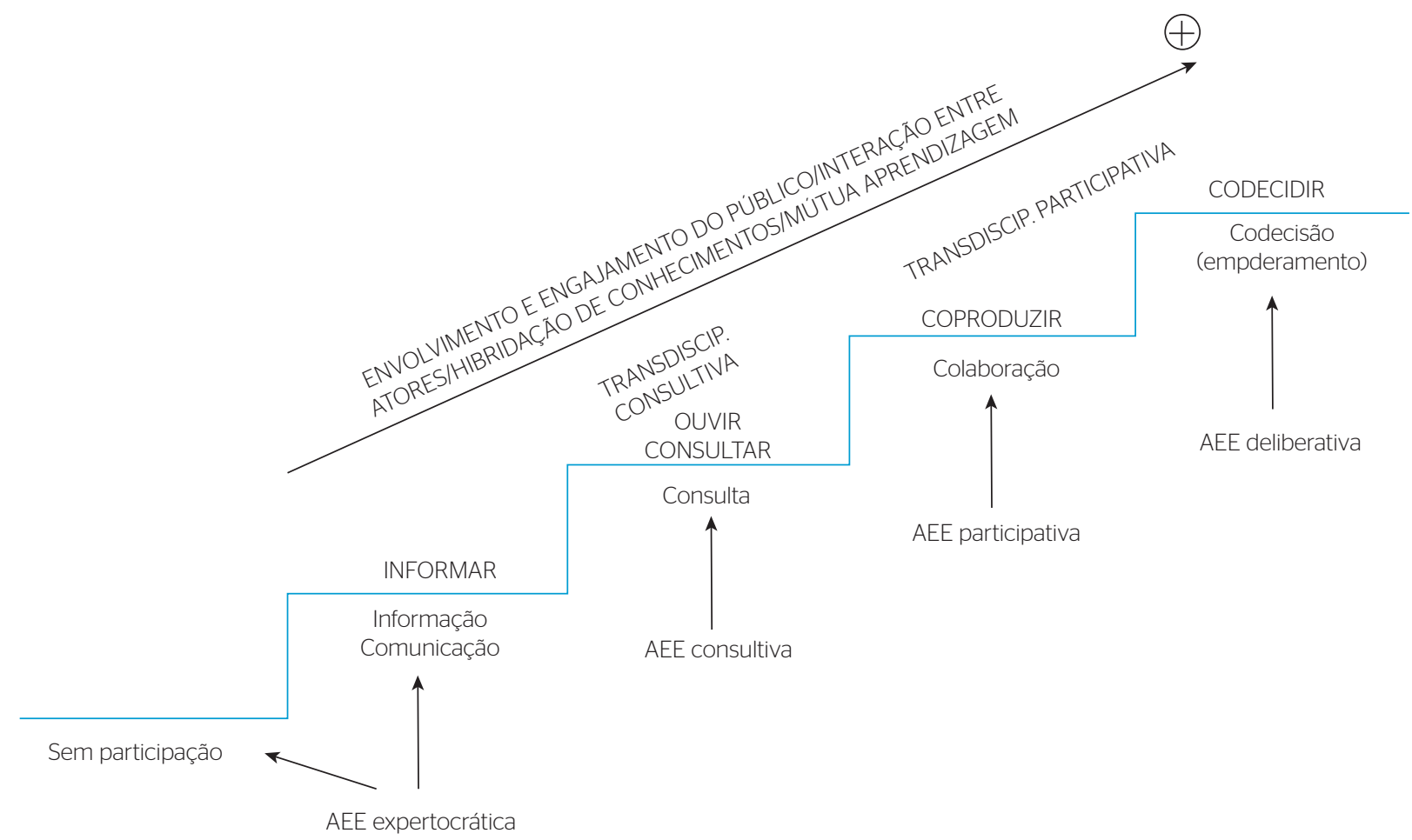

AAE: Avaliação Ambiental Estratégica.

Base: Bartlett e Oldgard (2003), Hage et al. (2010), Mobjörk (2010)

Figura 3 - Níveis de participação de atores leigos e sua relação com modelos de Avaliação Ambiental Estratégica e classes de transdisciplinaridade.

Além dos níveis de envolvimento e engajamento, outro fator condiciona a possibilidade de atuação de agentes leigos e, por conseguinte, de incorporação do CL: o grau de intervenção que lhes é facultado no processo. Hage et al. (2010) e Spengler (2009) demonstram que as atividades de participação do público interessado podem

1. limitar-se à transmissão passiva e unidirecional de informações ou comunicação das conclusões;

2. servir de meio para ouvir e consultar os atores sociais, recolhendo suas manifestações em questões a eles apresentadas;

3. propiciar efetiva colaboração dos envolvidos por meio de diálogo e negociação, resultando em coprodução entre ciência e CL; ou

4. além de coprodução, permitir que os participantes atuem como agentes de codecisão e deliberação acerca dos aspectos tratados na avaliação.

Bartlett e Oldgard (2003) associam esses níveis de participação a diferentes modelos de AAE, respectivamente expertocrática (essencialmente técnica e top-down), consultiva, participativa ou deliberativa, sendo os três últimos relacionados à concepção de Mobjörk (2010) referente à TD consultiva ou participativa (Figura 3).

Ainda que a atuação dos segmentos interessados possa alcançar o nível de codecisão, na maior parte das vezes o processo é limitado a momentos de informação ou de consulta (GAUTHIER et al., 2011; RAUSCHMAYER \& RISSE, 2005), normalmente realizados apenas ao final da AAE (BARTLETT \& OLDGARD, 2003), ocasião em que o público pode, no máximo, comentar as propostas. Assim, a possibilidade de oferecer novas visões, manifestar interesses e incorporar o CL é mais exceção do que regra (SHEATE \& PARTIDÁRIO, 2010).

\section{CONCLUSÕES}

A adoção de uma abordagem transdisciplinar poderá qualificar o processo de AAE. Para tal, a produção de conhecimento deve estar baseada na atuação interdisciplinar do corpo técnico e na inclusão do saber próprio da população envolvida. Para consolidar este segundo aspecto, é necessário viabilizar processos de participação colaborativa dos atores sociais, bem como adotar boas práticas que 
favoreçam a captura, o compartilhamento e a utilização do CL. Entre estas, cabe destacar:

- compor equipes de elaboração com a inclusão de membro(s) leigo(s), oriundo(s) da população afetada, para o papel de articulação com o público e a promoção do emprego do CL;

- empregar ampla variedade de mídias para divulgar o processo de AAE e para provocar e motivar o engajamento dos cidadãos;

- mapear, antecipadamente, as partes envolvidas e o público interessado, decidir formas de participação e colaboração (GAUTHIER et al., 2011) e dar publicidade a essas questões;

- garantir adequada representação dos vários segmentos sociais, pois o público é um grupo heterogêneo (CONNELLY \& RICHARDSON, 2005), o mesmo acontecendo com o CL;

- propiciar e estimular a participação dos interessados, já ao início do processo de avaliação e ao longo de toda a sua extensão (RAUSCHMAYER \& RISSE, 2005; REED, 2008);

- disponibilizar, em estágio precoce do processo, material de apoio técnico-informativo elaborado em nível e linguagem que permitam perfeita compreensão pelo público local;

- utilizar a maior diversidade possível de formas e métodos de participação e interação, incluindo processos presenciais e não presenciais (GONZÁLEZ et al., 2008);

- prover avenidas de comunicação e atualização entre os envolvidos, valendo-se, por exemplo, de portal eletrônico de conhecimento, exclusivo para a AAE em desenvolvimento;
- emitir informes curtos e sucessivos sobre o desenrolar do processo (PARTIDÁRIO, 2007), mantendo o público atualizado e alimentando seu interesse em participar;

- no caso de reuniões públicas, realizá-las em pequenos grupos ou por comunidade, de modo a evitar a inibição frente a grandes plateias, favorecendo, assim, a participação;

- fazer com que as atividades presenciais tenham uma correta distribuição no tempo (todas as fases da avaliação) e no espaço (área geográfica abrangida pela $\mathrm{AAE}$ );

- estimular o emprego de "corretores do conhecimento" (brokers), a fim de facilitar o contato e o intercâmbio de informações com o público (SHEATE \& PARTIDÁRIO, 2010);

- conceder particular atenção aos "especialistas locais", pessoas da comunidade que demonstrem excelência de conhecimento em um campo de saber relevante à $\mathrm{AAE}$;

- instituir que cada processo de AAE catalogue e divulgue as "lições aprendidas" quanto ao trabalho cooperativo com a população e ao emprego de CL nos respectivos estudos;

- formar comunidades de prática envolvendo elaboradores, pesquisadores e outros interessados em AAE, visando troca de conhecimentos e compartilhamento de experiências.

Ressalta-se, por fim, que a adoção da abordagem transdisciplinar poderá imprimir um novo sentido à $\mathrm{AAE}$, ao destacar a importância não apenas do capital natural, mas também - e especialmente - do capital intelectual e do capital social que integram o contexto em estudo.

\section{REFERÊNCIAS}

AZCARATE, J. \& BALFORS, B. (2009) Participative SEA approach for data collection and objective formulation. Journal of Environmental Assessment Policy and Management, v. 11, n. 2, p. 189-211.

BALDUCCI, A.; CALVARESI, C.; ZIMMERMANN, K. (2011) Strategic environmental assessment, strategic spatial planning and the politics of local knowledge. In: ATKINSON, R.; TERIZAKIS, G.; ZIMMERMANN, K. (Eds.). Sustainability in European environmental policy: challenges of governance and knowledge. London: Routledge. p. 131-146.

BARTLETT, S.; OLDGARD, M. Expertocratic, consultative, participative or deliberative SEA? Toward guidelines for determining an appropriate level of public involvement in SEA. In: Easy-Eco2 Conference, Vienna, 2003. Proceedings... Disponível em: <http://www.wu.ac.at/inst/fsnu/ easy2/papers/a2_oldgard.pdf>. Acesso em: 28 jul. 2011.

BOND, A.J.; VIEGAS, C.V.; COELHO, C.C.S.R.; SELIG, P.M. (2O10) Informal knowledge processes: the underpinning for sustainability outcomes in EIA? Journal of Cleaner Production, v. 18, p. 6-13.
BONIFAZI, A.; REGA, C.; GAZZOLA, P. (2011) Strategic Environmental Assessment and the democratisation of spatial planning. Journal of Environmental Assessment Policy and Management, v. 13, n. 1 , p. 9-37.

BOOTH, A. \& SKELTON, N.W. (2011) "We are fighting for ourselves" - First Nations' evaluation of British Columbia and Canadian environmental assessment processes. Journal of Environmental Assessment Policy and Management, v. 13, n. 3, p. 367-404.

CASHMORE, M. (2004) The role of science in environmental impact assessment: process and procedure versus purpose in the development of theory. Environmental Impact Assessment Review, v. 24, p. 403-426.

CONNELLY, S. \& RICHARDSON, T. (2005) Value-driven SEA: time for an environmental justice perspective? Environmental Impact Assessment Review, v. 25, p. 391-409. 
CROAL, P.; TETREAULT, C.; e membros da IAIA Secção IP. (2012) Respecting indigenous peoples and traditional knowledge. Fargo, USA: International Association for Impact Assessment. Special Publication Series N. 9.4 p.

ELLIS, S.C. (2005) Meaningful consideration? A review of traditional knowledge in environmental decision making. Arctic, v. 58, n. 1, p. 66-77.

FAILING, L.; GREGORY, R.; HARSTONE, M. (2007) Integrating science and local knowledge in environmental risk management: A decisionfocused approach. Ecological Economics, v. 64, p. 47-60.

FISCHER, T.B. (2005) Having an impact? Context elements for effective SEA application in transport policy, plan and programme making. Journal of Environmental Assessment Policy and Management, v. 7, n. 3. p. 407-432

GAUTHIER, M.; SIMARD, L.; WAAUB, J.P. (2011) Public participation in strategic environmental assessment (SEA): Critical review and the Quebec (Canada) approach. Environmental Impact Assessment Review, v. 31, p. 48-60.

GONZÁLEZ, A.; GILMER, A.; FOLEY, R.; SWEENEY, J.; FRY, J. (2008) Technology-aided participative methods in environmental assessment: An international perspective. Computers, Environment and Urban Systems, v. 32, p. 303-316.

HAGE, M.; LEROY, P.; PETERSEN, A.C. (2010) Stakeholder participation in environmental knowledge production. Futures, v. 42, p. 254-264.

ICSU - INTERNATIONAL COUNCIL FOR SCIENCE. (2002) Science, traditional knowledge and sustainable development. Paris, France: ICSU. 24 p. (ICSU Series on Science for Sustainable Development, 4).

KWIATKOWSKI, R.E.; TIKHONOV, C.; PEACE, D.M.; BOURASSA, C. (2009) Canadian Indigenous engagement and capacity building in health impact assessment. Impact Assessment and Project Appraisal, v. 27, n. 1, p. 57-67.

LUKS, F. \& SIEBENHÜNER, B. (2007) Transdisciplinarity for social learning? The contribution of the German socio-ecological research initiative to sustainability governance. Ecological Economics, v. 63, p. $418-426$.

MARCONI, M.A. \& LAKATOS, E.M. (2010) Fundamentos de metodologia científica. 7 ed. São Paulo: Atlas. 297 p.

MOBJÖRK, M. (2010) Consulting versus participatory transdisciplinarity: a refined classification of transdisciplinary research. Futures, v. 42, p. 866-873.

MOREIRA, H. \& CALEFFE, L.G. (2008) Metodologia da pesquisa para o professor pesquisador. 2 ed. Rio de Janeiro: Lamparina.

NILSSON, M.; BJÖRKLUND, A.; FINNVEDEN, G.; JOHANSSON, J. (2005) Testing a SEA methodology for the energy sector: a waste incineration tax proposal. Environmental Impact Assessment Review, v. 25, p. 1-32

O'FAIRCHEALLAIGH, C. (2007) Environmental agreements, EIA followup and aboriginal participation in environmental management: The Canadian experience. Environmental Impact Assessment Review, v. 27, p. 319-342.
PACl, C.; TOBIN, A.; ROBB, P. (2002) Reconsidering the Canadian Environmental Impact Assessment Act: A place for traditional environmental knowledge. Environmental Impact Assessment Review, v. 22, p. 111-127.

PARTIDÁRIO, M.R. (2000) Elements of an SEA framework - improving the added-value of SEA. Environmental Impact Assessment Review, v. 20, p. 647-663.

PARTIDÁRIO, M.R. (2007) Guia de boas práticas para Avaliação Ambiental Estratégica: orientações metodológicas. Amadora: Agência Portuguesa do Ambiente. 63 p.

POHL, C. (2008) From science to policy through transdisciplinary research. Environmental Science and Policy, v. 11, p. 46-53.

RAUSCHMAYER, F. \& RISSE, N. (2005) A framework for the selection of participatory approaches for SEA. Environmental Impact Assessment Review, v. 25, p. 650-666

REED, M.S. (2008) Stakeholder participation for environmental management: A literature review. Biological Conservation, v. 141 p. 2417-2431.

RUNHAAR, H. (2009) Putting SEA in context: a discourse perspective on how SEA contributes to decision-making. Impact Assessment and Project Appraisal, v. 29, p. 200-209.

RUNHAAR, H. \& DRIESSEN, P.P.J. (2007) What makes strategic environmental assessment successful environmental assessment? The role of context in the contribution of SEA to decision-making Impact Assessment and Project Appraisal, v. 25, n. 1, p. 2-14

SALLENAVE, J. (1994) Giving traditional ecological knowledge its rightful place in environmental impact assessment. Northern Perspectives, v. 22, n. 1, p. 16-19.

SHEATE, W.R. \& PARTIDARIO, M.R. (2010) Strategic approaches and assessment techniques - Potential for knowledge brokerage towards sustainability. Environmental Impact Assessment Review, v. 30, p. $278-288$

SPENGLER, L. (2009) Public participation in Strategic Environmental Assessment in China. Wuppertal: Wuppertal Institute. 33 p.

STEVENSON, M.G. (1996) Indigenous knowledge in environmental assessment. Arctic, v. 49, n. 3, p. 278-291.

STOCK, P. \& BURTON, R.J.F. (2011) Defining terms for integrated (multi-inter-trans-disciplinary) sustainability research. Sustainability, v. 3, p. 1090-1113.

STOEGLEHNER, G.; BROWN, A.L.; KØRNØV, L.B. (2009) SEA and planning: "ownership" of strategic environmental assessment by the planners is the key to its effectiveness. Impact Assessment and Project Appraisal, v. 27, n. 2, p. 111-120

THERIVEL, R. (2010) Strategic Environmental Assessment in action. 2 ed. London: Earthscan. 366 p.

TRESS, G.; TRESS, B.; FRY, G. (2004) Clarifying integrative research concepts in landscape ecology. Landscape Ecology, v. 20, p. 479-493. 
UITERKAMP, A.J.M.S. \& VLEK, C. (2007) Practice and outcomes of multidisciplinary research for environmental sustainability. Journal of Social Issues, v. 63, n. 1, p. 175-197.

USHER, P.J. (2000) Traditional Ecological knowledge in environmental assessment and management. Arctic, v. 53, n. 2, p. 183-193.

VAN BUUREN, A. \& NOOTEBOOM, S. (2009) Evaluating strategic environmental assessment in The Netherlands: content, process and procedure as indissoluble criteria for effectiveness. Impact Assessment and Project Appraisal, v. 27, n. 2, p. 145-154.

VAN BUUREN, A. \& NOOTEBOOM, S. (2010) The succesS of SEA in the Ducth planning practice: how formal assessment can contribute to collaborative governance. Environmental Impact Assessment Review, v. 30, p. 127-135.
VAN DOREN, D;; DRIESSEN, P.P.J.; SCHIJF, B.; RUNHAAR, H.A.C. (2O13) Evaluating the substantive effectiveness of SEA: Towards a better understanding. Environmental Impact Assessment Review, v. 38, p. 120-130.

WHITFIELD, S.; GEIST, H.J.; IORIS, A.A.R. (2011) Deliberative assessment in complex socioecological systems: recommendations for environmental assessment in drylands. Environmental Monitoring and Assessment, v. 183, p. 465-483.

WIEK, A.; BINDER, C. (2005) Solution spaces for decision-making - a sustainability assessment tool for city-regions. Environmental Impact Assessment Review, v. 25, p. 589-608.

YLI-PELKONEN, V.; KOHL, J. (2005) The role of local ecological knowledge in sustainable urban planning: perspectives from Finland. Sustainability: Science, Practice, \& Policy, v. 1, n. 1, p. 3-14. 